\title{
TEM Based High Resolution Electron Diffraction Techniques for Three- dimensional Nanostructure Determination
}

Jian-Min Zuo ${ }^{1,2}$, Yifei Meng ${ }^{1,2}$, Piyush Vivek Deshpande ${ }^{1,2}$, Yang $\mathrm{Hu}^{1,2}$, Kyou-Hyun $\mathrm{Kim}^{1,2}$, Hui Xing ${ }^{1,2,3}$, Peng Zhang ${ }^{4}$ and Haifeng $\mathrm{Wang}^{4}$

${ }^{1}$ Dept of Materials Science and Engineering, University of Illinois, Urbana-Champaign, IL 61801

${ }^{2}$ Frederick Seitz Materials Research Laboratory, University of Illinois, Urbana-Champaign, IL 618013

${ }^{3}$ School of Materials Science and Engineering, Shanghai Jiaotong University, China

${ }^{4}$ Western Digital Technologies, Inc., 44200 Osgood Road, Fremont CA, 94539

Many technologically important materials possess complex nanostructures and structure dependent properties. Transmission electron diffraction (TED) is an appropriate technique for complex nanostructure analysis because it is highly sensitive to local structure and it can be obtained using a small electron beam [1-4]. Compared to the scanning electron microscope (SEM) based electron backscattering diffraction technique, the small interaction volume in TED allows for high spatial resolution.

Traditionally, TED is performed either by using parallel beam illumination with the help of a selected area aperture for selected area electron diffraction (SAED) or by using a focused beam for convergent beam electron diffraction (CBED). Electron nanodiffraction can be performed in a modern TEM instrument using a parallel electron beam of nanometers in diameter with help of an additional condenser lens (minilens) [3, 4]. For complex nanostructure analysis, there is a need to record multiple diffraction patterns by scanning from desired sample areas. Two major approaches have been developed thus far, one uses the built-in STEM scan coil and performs electron nanodiffraction in STEM mode, and the other uses external scan and precession driver coupled with an external camera[5]. For example, Alloyeau et al. [6] and Ganesh et al. [7] have reported the use of a small probe controlled by the Gatan ${ }^{\mathrm{TM}}$ software "STEM Diffraction Imaging" to acquire diffraction patterns on a pixel by pixel basis. For three-dimensional microstructure determination, EBSD coupled with FIB cross-section or nondestructive X-ray diffraction techniques have been developed. More recently, Liu et al. reported a TEM based technique using conical scan [8].

Here, we report on a TEM based scanning electron nanodiffraction (SEND) technique that uses the built-in TEM deflection coils. Diffraction pattern recording and beam scanning are automated using a DigitalMicrograph $^{\circledR}$ script to control the TEM deflection coils and camera readout [9]. In a conventional TEM with $\mathrm{LaB}_{6}$ source, SEND can be performed in low dose mode using electron beams of 2 5 $\mathrm{nm}$ in full-width at half-maximum (FWHM) and $0.1 \mathrm{pA}$ or less in beam current. Electron diffraction pattern indexing is achieved by a combination of correlation analysis of the recorded diffraction patterns, diffraction peak search and peak indexing using both length and angle information. We demonstrate the performance of this approach nanostructure determination in several nanostructure materials (see Figure 1 for an example). By coupling with a tomographic holder, SEND can be performed with large sample tilts and thus the opportunity for the determination of three-dimensional domains at nanometer resolution [10].

References:

[1] Cowley, J.M., "Electron Diffraction Techniques." 1993: International Union of Crystallography. 
[2] Spence, J.C.H. and J.M. Zuo, "Electron microdiffraction". 1992: Plenum Press.

[3] Zuo, J.M. et al, Science, 2003. 300(5624): p. 1419-1421.

[4] Zuo, J.M. et al. Microscopy Research and Technique, 2004. 64(5-6): p. 347-355.

[5] Rauch, E.F. et al, Zeitschrift Fur Kristallographie, 2010. 225(2-3): p. 103-109.

[6] Alloyeau, D., et al, Ultramicroscopy, 2008. 108(7): p. 656-662.

[7] Ganesh, K., et al, Microscopy and Microanalysis, 2010. 16(SupplementS2): p. 1728-1729.

[8] Liu, H.H., et al, Science, 2011. 332(6031): p. 833-834.

[9] Tao, J., et al, Physical Review Letters, 2009. 103(9).

[10] The work reported here is supported by DOE BES under contract DEFG02-01ER45923 and a grant from Western Digital.

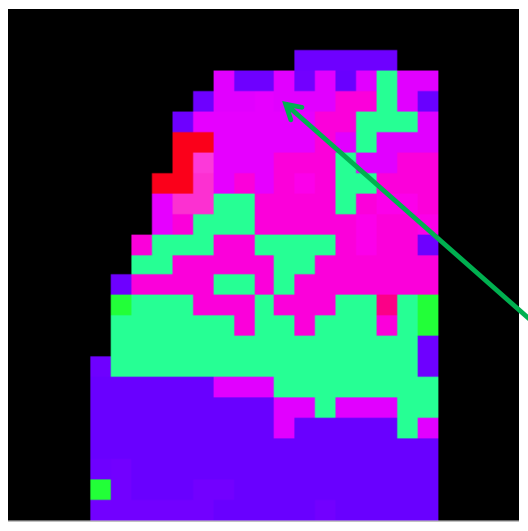

[001] [011]
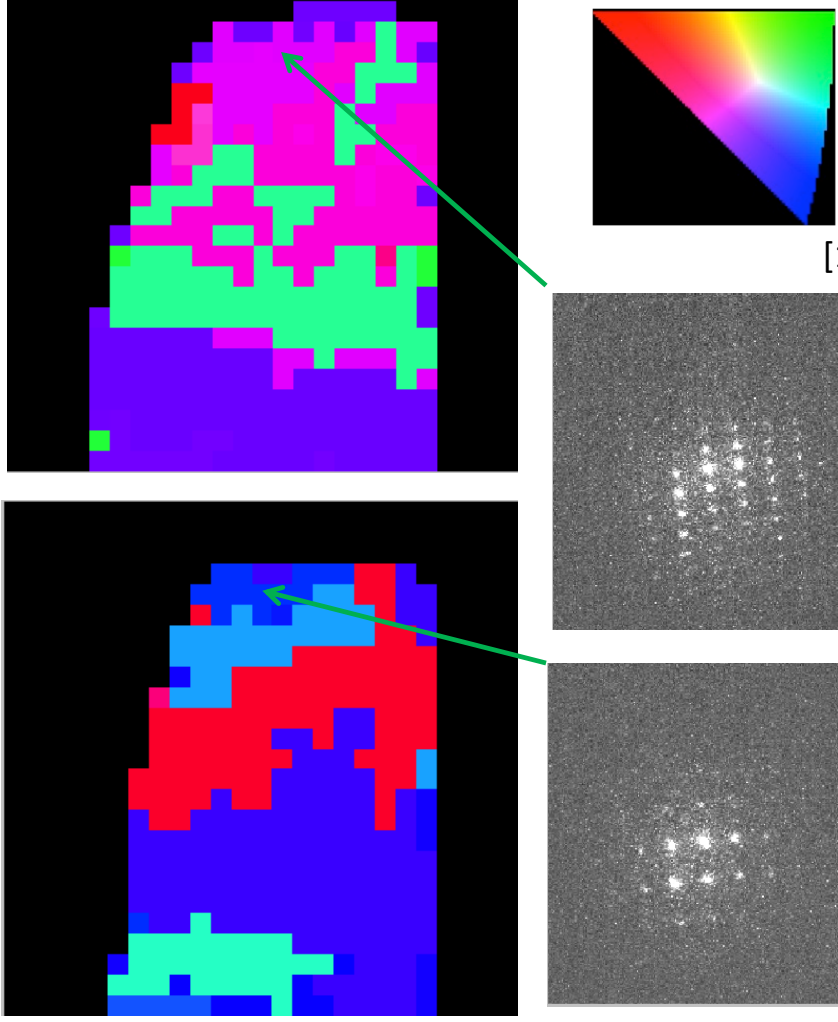

[111]
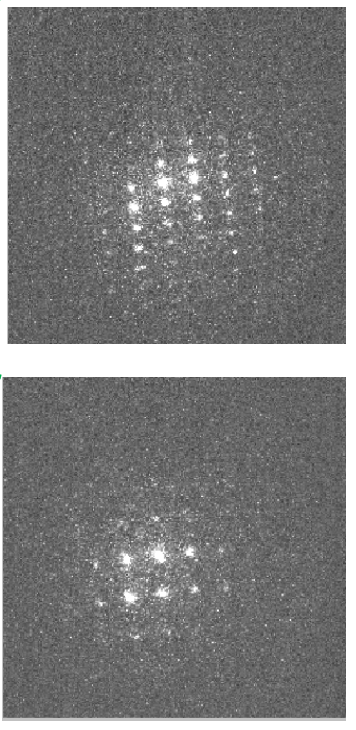

Figure 1. Orientation maps obtained from a high entropy alloy $\left(\mathrm{Al}_{0.5} \mathrm{CoCrCuFeNi}\right)$ at two orientations of 30 degrees apart. The sample was first fabricated into a needle shape using FIB. SEND was performed using $25 \times 25$ sampling points over $240 \times 240 \mathrm{~nm}^{2}$ using an electron probe of $8 \mathrm{~nm}$ in FWHM The diffraction patterns were indexed using software developed at University of Illinois and colored according to the inverse pole figure at top-right corner. Two individual experimental diffraction patterns are shown on left. 